\title{
Dynamic Analysis of Planetary Gear Train Based on ADAMS
}

\author{
Yongliang Yuan \\ School of mechanical engineering, Dalian University of Technology, Dalian, China
}

\begin{abstract}
Automatic pin machine is a kind of typical equipment widely used in mechanical and electronic industry, which is mainly used for automatic assembly of pinhole parts. In order to improve the efficiency and stability of the transmission system, the dynamic performance of planetary gears is studied. This paper uses UG to establish the dynamic model of planetary gears. Dynamic simulation and its motion law are obtained using Adams. The contact force between the planet gear and the center gear fluctuates around $5000 \mathrm{~N}$, and increases with the load. The results show that Adams can provide an effective prediction of the dynamic characteristics of planetary gear trains, and have a certain reference value for future structural improvements.
\end{abstract}

\section{Introduction}

Automatic pin machine is a kind of typical equipment widely used in mechanical and electronic industry, which is mainly used for automatic assembly of pinhole parts. The dynamic performance of the pin machine will directly affect the assembly efficiency and assembly accuracy. In order to improve the efficient and stable movement of mechanical equipment, many researchers have focused on kinematics analysis.

With the development of computer technology, virtual prototyping has become one of the necessary tools for researchers. Virtual prototyping technology not only predict the dynamic performance of the equipment, but also quickly improve the product structure and shorten the research and development cycle.

To improve the comprehensive performance of an automatic pin machine, dynamic performance of the transmission system has been analyzed in this paper. The dynamics model is established by UG and the simulation analysis is carried out by using the mechanical dynamics simulation software Adams. The dynamic performance of the planetary gear train is obtained, which lays a foundation for the optimization design of the mechanism in the future.

\section{The working principle of the model}

Planetary gear train is a swing gear train with only one degree of freedom. The planetary gear train is a kind of coaxial transmission, and several identical planetary gears are distributed around the sun gear. The planetary gear train has the advantages of compact structure, small volume, small mass, large carrying capacity, large transmission power range and transmission range, low running noise, high efficiency and long life.
The planetary gear train used in the automatic pin machine is composed of one sun gear, five planetary gears and one fixed gear, the structure is shown in figure 1.

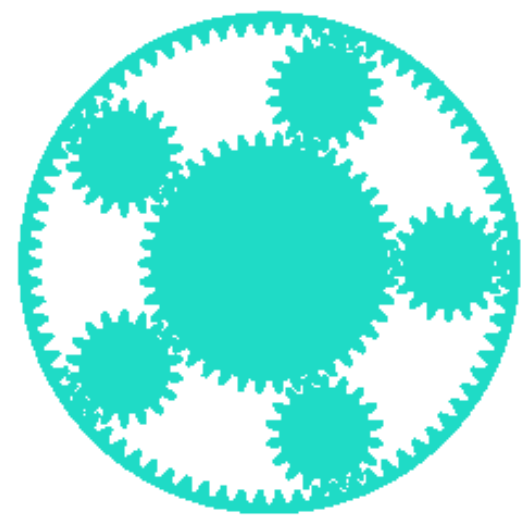

Figure 1. Schematic diagram of planetary gear train structure.

The sun gear rotates and transmits the power to the five planetary gears by engaging with the five planetary gears. Finally, the rotation of the five planetary gears leads to the rotation of the automatic fixture platform to realize the rotation of the work piece, and then to install the pinhole parts into the connector and other work pieces under the work of the automatic manipulator.

\section{Meshing curve calculation}

The meshing line of a gear is very complicated, and the accuracy of the meshing line is related to the transmission accuracy of the gear system. The meshing of the gear is shown in figure 2 . 


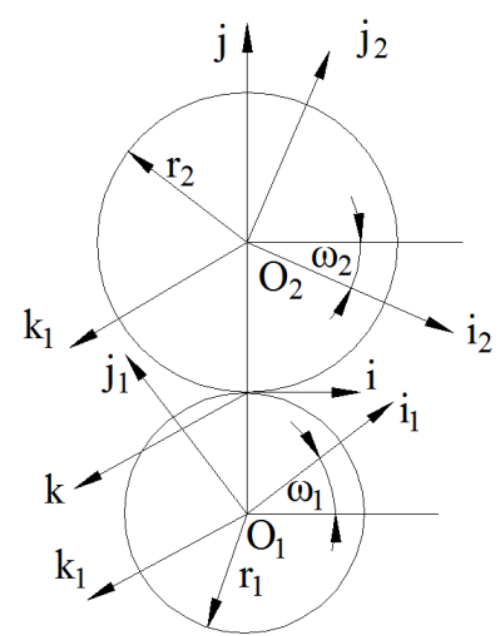

Figure 2. Gear meshing diagram.

The meshing point coordinates on gear 1 is given by: $\boldsymbol{r}_{1}=x_{1}(u) \boldsymbol{i}_{1}+y_{1}(u) \boldsymbol{j}_{1}$

$$
=\left(r_{b 1} \sin u-u r_{b 1} \cos u\right) \boldsymbol{i}+\left(r_{b 1} \cos u+u r_{b 1} \sin u\right) \boldsymbol{j}
$$

The meshing point coordinates on gear 1 is given by: $\mathbf{v}_{12}=\omega_{12} \times \mathbf{r} \mathbf{1}+\omega_{2} \times \mathbf{a}$

$$
\begin{aligned}
& =\left(1+1 / i_{12}\right) \mathbf{k}_{1} \times\left(x_{1} \mathbf{i}_{1}+y_{1} \mathbf{j}_{1}\right)-1 / i_{12} \mathbf{k}_{1} \times a\left(\sin \varphi_{1} \mathbf{i}+\cos \varphi_{1} \mathbf{j}\right) \\
& \left.=\left[-\left(1+1 / i_{12}\right) y_{1}+1 / i_{12} a \cos \varphi_{1}\right)\right] \mathbf{i}_{1}+\left[\left(1+1 / i_{12}\right) x_{1}+1 / i_{12} a \sin \varphi_{1}\right] \mathbf{j}_{1}
\end{aligned}
$$

where

$$
a=O_{1} O_{2}=a j=a\left(\sin \varphi_{1} i+\cos \varphi_{1} j\right)
$$

$i_{12}$ is the transmission ratio;

$r_{1}$ is the radius of the driving gear;

$r_{2}$ is the radius of the driven gear;

$a$ is the center-to-center distance;

$\varphi_{1}$ is angle speed of the driving gear;

$\varphi_{2}$ is angle speed of the driving gear.

Meshing equation is given by:

$\cos \left(u+\varphi_{1}\right)=\left(y_{1} \cos u-x_{1} \sin u\right)\left(1+i_{12}\right) / a$

$\varphi_{1}=a \cos \left(\left(y_{1} \cos u-x_{1} \sin u\right)\left(1+i_{12}\right) / a\right)-u$

\section{Dynamic simulation analysis}

Adams is a multibody dynamics simulation software for MSC company. Based on Lagrangian equation method, the system dynamics equation is established, and the virtual kinematics and dynamics analysis of a mechanical system is carried out. Finally, the motion law has been obtained ${ }^{[5-6]}$.

\subsection{Establishment of dynamic model}

There are many methods to build dynamics model. Because the involute of gear model is more complicated, and it is difficult to establish the model, this paper uses UG software to build planetary gear train model. The established planetary gear train model was imported into Adams, and the model was established ${ }^{[7]}$.

\subsection{Constraints}

The model is a dynamic model of a planetary gear train. Due to the model is full of gears, the main constraints used in this paper are revolution joint and contact. All gears in the model are rotated with the ground, and the center of rotation is at the center of the gear. Contact constraints should be added between the center gear and the five planetary gears, and friction should be added to the contact setting. The constraint between the fixed gear and the five planetary gears is the same as the parameter between the center gear. The model with the added constraint is shown in figure 2 .

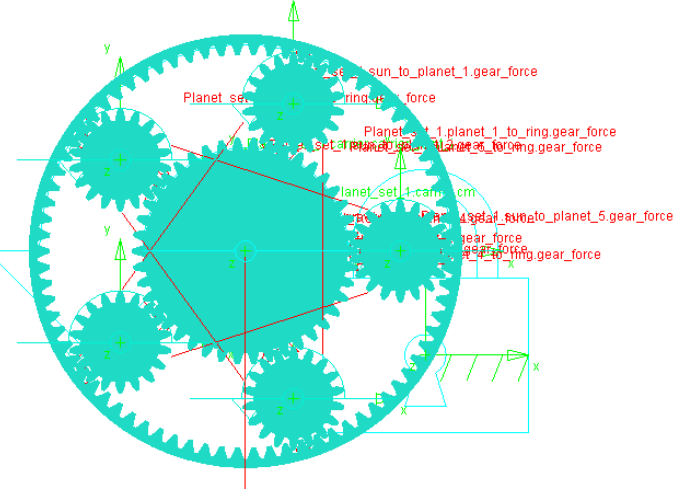

Figure 2. Constrained planetary gear train.

\subsection{Software implementations}

In order to obtain the dynamic characteristics of the planetary gear train, the simulation of different working conditions is carried out in this paper, and the dynamic characteristic curve of the planetary gear train is obtained by comparison.

Case 1: planetary gear train without load

Firstly, the dynamics simulation analysis of the planetary gear train is verified the dynamic performance of the planetary gear train. The displacement velocity of the planetary gears during meshing is shown in Figure 3.

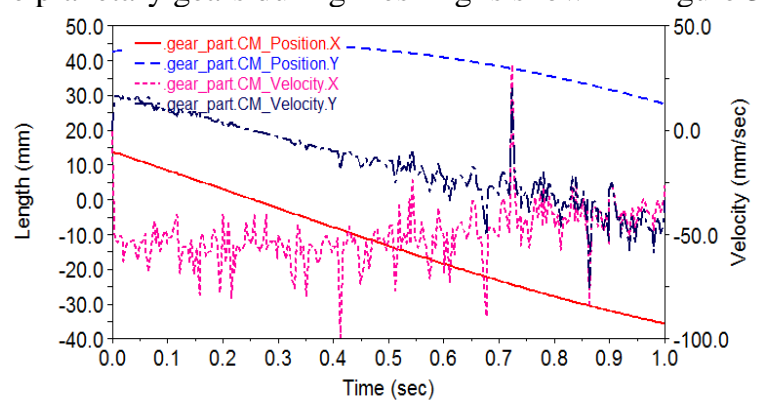

Figure 3. Displacement velocity of planetary gears during meshing.

Table 1. Displacement velocity of planetary gears during meshing.

\begin{tabular}{ccc}
\hline & Maximum & Minimum \\
\hline X_displacement & $14.7 \mathrm{~mm}$ & $-33.7 \mathrm{~mm}$ \\
Y_displacement & $42.9 \mathrm{~mm}$ & $28.3 \mathrm{~mm}$ \\
X_speed & $26.1 \mathrm{~mm} / \mathrm{s}$ & $-99.2 \mathrm{~mm} / \mathrm{s}$ \\
Y_speed & $24.9 \mathrm{~mm} / \mathrm{s}$ & $-61.5 \mathrm{~mm} / \mathrm{s}$ \\
\hline
\end{tabular}

As can be seen from Figure 3, the displacement of the planet gears is from $-50 \mathrm{~mm}$ to $50 \mathrm{~mm}$, which is caused mainly by the limitation of fixed gear on the planet gears. 
By analyzing the velocity in $\mathrm{X}$ and $\mathrm{Y}$ directions, we know that the fluctuation of velocity in $X$ direction is more obvious than in $\mathrm{Y}$ direction. The main reason for this phenomenon is the gap between gears. To improve the smoothness of planetary gear transmission, the clearance between gears should be properly lubricated.

The contact force between the planetary gear and the sun gear is shown in Figure 4.

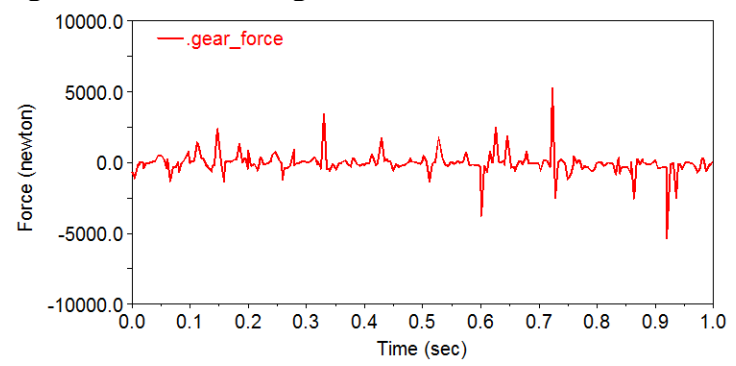

Figure 4. Contact force between planetary gear and sun gear.

Figure 4 shows that the contact force between the planetary gear and the sun gear fluctuates within $5000 \mathrm{~N}$, with a maximum value of $5293.7 \mathrm{~N}$. This phenomenon is influenced by the time-varying meshing stiffness and clearance of gears. In order to reduce wear, it is very important to increase lubrication between gears.

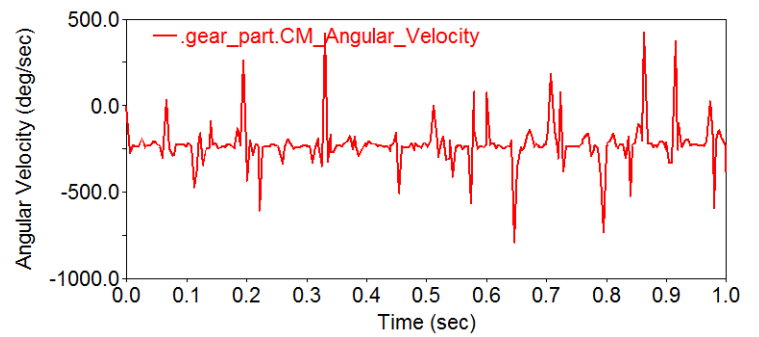

Figure 5. The angular velocity of the planetary gears.

Figure 5 shows that the angular velocity of planetary gears fluctuates around $400^{\circ} / \mathrm{s}$. There are several peaks and valleys in the figure, which shows that the planetary gear does not work smoothly, and there is a certain vibration.

Case 2: planetary gear train with $100 \mathrm{~N}$ load

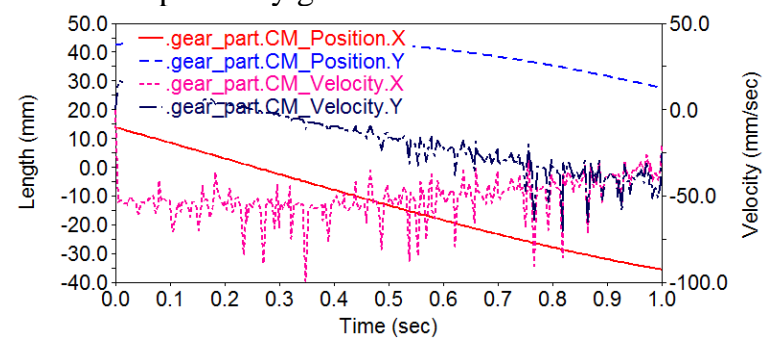

Figure 6. Displacement velocity of planetary gears during meshing.

Table 2. Displacement velocity of planetary gears during meshing.

\begin{tabular}{ccc}
\hline & Maximum & Minimum \\
\hline X_displacement & $14.7 \mathrm{~mm}$ & $-33.7 \mathrm{~mm}$ \\
Y_displacement & $42.9 \mathrm{~mm}$ & $28.4 \mathrm{~mm}$ \\
X_speed & $20.4 \mathrm{~mm} / \mathrm{s}$ & $-99.1 \mathrm{~mm} / \mathrm{s}$ \\
Y_speed & $24.9 \mathrm{~mm} / \mathrm{s}$ & $-57.8 \mathrm{~mm} / \mathrm{s}$ \\
\hline
\end{tabular}

Compared with figure 3 , the fluctuation of $\mathrm{X}$ direction in figure 6 is significantly lower than the maximum velocity in $\mathrm{X}$ direction of figure 3 is $99.2 \mathrm{~mm} /$ $\mathrm{s}$, and the maximum error is within $0.5 \%$. With the increase of load, the fluctuation of $\mathrm{X}$ direction slows down, which accords with the phenomenon of low speed and heavy load. The velocity in the $\mathrm{Y}$ direction is similar to that in the $\mathrm{X}$ direction, with a maximum value of 70.1 $\mathrm{mm} / \mathrm{s}$.

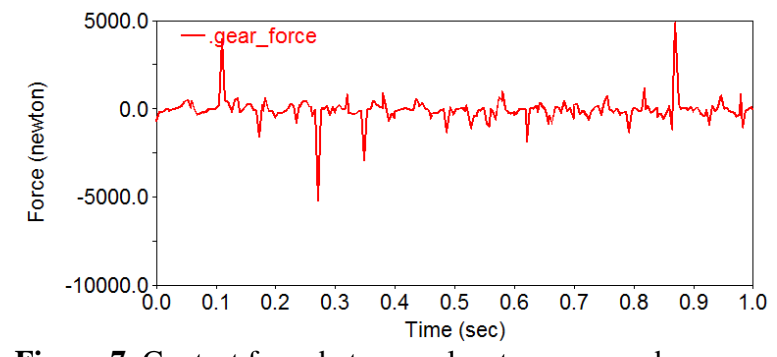

Figure 7. Contact force between planetary gear and sun gear.

Figure 7 shows that the contact force between the planetary gear and the sun gear fluctuates within $5000 \mathrm{~N}$, with a maximum value of 5310.3N. Compared with Figure 4, the fluctuation of the contact force is obviously smaller than that of without load in the case of a load, and the maximum value changes little compared with Figure 4 , and the variation is about $20 \mathrm{~N}$. The results show that the contact force increases with the increase of the load, and the contact force is not affected because the load is small when the pin machine is working.

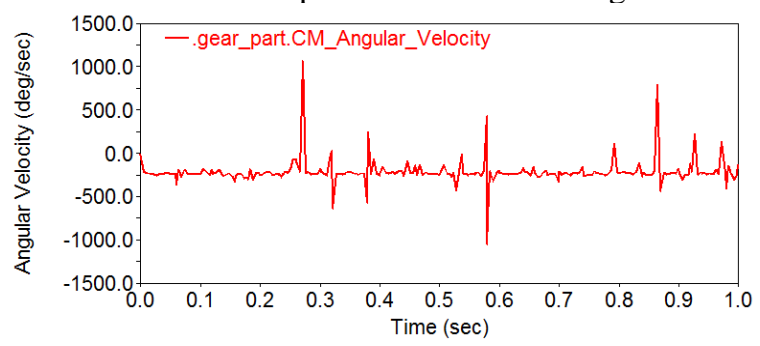

Figure 8. The angular velocity of the planetary gears.

Figure 8 shows that the angular velocity of planetary gears fluctuates around $300^{\circ} / \mathrm{s}$. Compared to figure 5, the range of fluctuations is smaller, but the peak is larger. The main reason is that there is vibration in the transmission process, and under the condition of load. There is a continuous starting phenomenon.

\section{Conclusion}

Through the analysis of the working principle of the pin machine, planetary gear train is established using UG.Then the calculation of the meshing curve has carried on the theoretical analysis. In this paper, Adams is used for simulation analysis, and the dynamic characteristics of different working conditions are analyzed.The motion law and dynamics curve are obtained, which has certain reference value for the later optimization design. The results show that the Adams can effectively verify the model simulation test, not only can get the law of motion, but also can effectively prevent its failure. 


\section{References}

1. Q . Tian, C. Liu, L.K. Liu. J. Sys. Simulation. 257 (2013).

2. J.L. Rong, Y.T. Yang, J. Li. J. As. 3311 (2012).

3. M.H. Vu, U.J. Na. IEEE T. Instrum. Meas. 6011 (2011).

4. K Nonami, R Yuasa, D Waterman. Auton. Robot. 18 3 (2005).

5. Y.L. Yuan, Z. Yang, S.H. Wang. Packing Eng. 35 11 (2014).

6. F. Qiu, Z. Yang, Y.L. Yuan. Packing Eng. 3614 (2015).

7. L.P. Chen, Y.L. Wang. More rigid body dynamics. Harbin engineering university press. (1995). 